Document downloaded from:

http://hdl.handle.net/10251/52700

This paper must be cited as:

Naranjo Ornedo, V.; Lloréns Rodríguez, R.; Alcañiz Raya, ML.; López-Mir, F. (2011). Metal artifact reduction in dental CT images using polar mathematical morphology. Computer Methods and Programs in Biomedicine. 102(1):64-74. doi:10.1016/j.cmpb.2010.11.009.

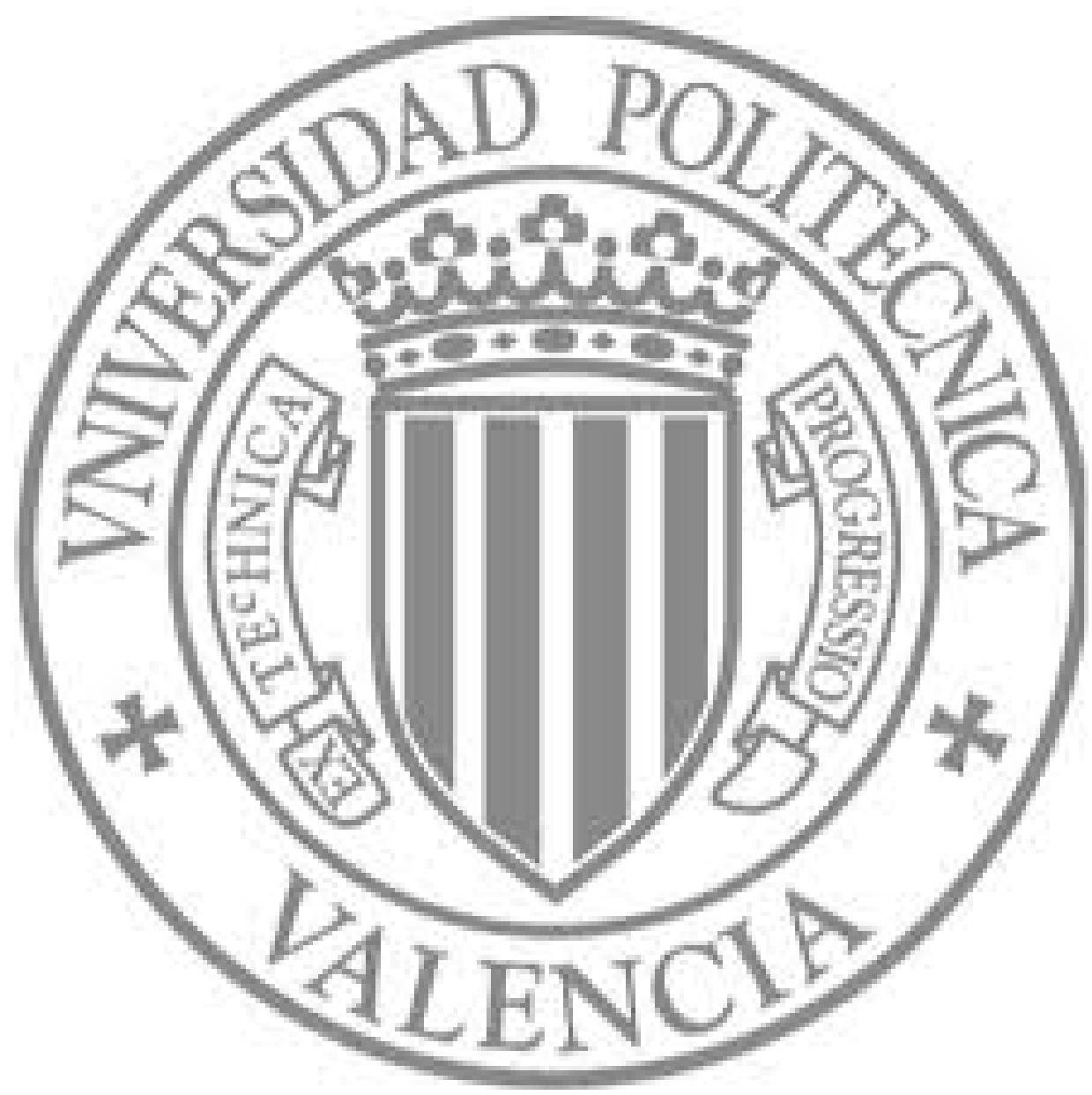

The final publication is available at

http://dx.doi.org/10.1016/j.cmpb.2010.11.009

Copyright Elsevier 


\title{
Metal Artifact Reduction in Dental CT Images using Polar Mathematical Morphology
}

\author{
V. Naranjo, R. Lloréns, M. Alcañiz and F. López \\ LabHuman \\ Universidad Politécnica de Valencia \\ Camino de Vera $s / n$ \\ 46022 Valencia (Spain) \\ vnaranjo@dcom.upv.es
}

\begin{abstract}
Most dental implant planning systems use a 3D representation of the CT scan of the patient under study as it provides a more intuitive view of the human jaw. The presence of metallic objects in human jaws, such as amalgam or gold fillings, provokes several artifacts like streaking and beam hardening which makes the reconstruction process difficult. In order to reduce these artifacts, several methods have been proposed using the raw data, directly obtained from the tomographs, in different ways. However, in DICOM-based applications this information is not available, and thus the need of a new method that handles this task in the DICOM domain. The presented method performs a morphological filtering in the polar domain yielding output images less affected by artifacts (even in cases of multiple metallic objects) without causing significant smoothing of the anatomic structures, which allows a great improvement in the $3 \mathrm{D}$ reconstruction. The algorithm has been automated and compared to other image denoising methods with successful results.
\end{abstract}

Keywords: Dental CT, Artifact reduction, Polar morphology

\section{Introduction}

The 3D representation of CT scans is widely used in medical applications such as virtual endoscopy, plastic reconstructive surgery, dental implant planning systems and more. This representation is built thresholding the axial CT slices which constitute the volume of the CT scan. 


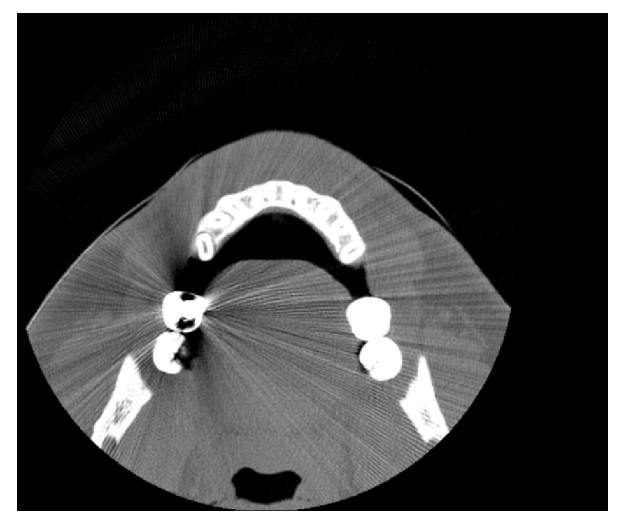

Figure 1: Example of MAR artifacts in a CT image from a jaw.

Metallic objects present in CT scans cause strong artifacts like beam hardening and streaking, strongly noticeable in axial images after being reconstructed by means of filtered back projection algorithm (FBP), as shown in figure 1. Metal artifacts complicate to a large extent the 3D reconstruction (figure 15).

FBP is the most extended method to reconstruct images from projections. The algorithm assumes that the tomography data is the Radon transform of the attenuation coefficients of the scanned objects. This assumption is plausible if the density of the objects is similar. But when objects with different densities, such as cavities $(\sim 200 \mathrm{HU})$, bones $(\sim 1200-1800 \mathrm{HU})$, teeth $(\sim 2000 \mathrm{HU})$ and dental fillings (temporary fillings: $\sim 6000-8500$ HU, composite fillings: $\sim 4500-17000 \mathrm{HU}$ and amalgam and gold $>30700$ $\mathrm{HU})$, are present at the same time, the reconstructed images have perceptible artifacts as streaking (high level rays emerging from metallic objects), beam hardening (shadows cast over the areas surrounding the object) and some other effects. Figure 1 shows a CT image of a jaw clearly affected by the previously described artifacts. Consequently, several research studies have tried to reduce these artifacts, and have approached the problem in different ways.

Most metal artifact reduction (MAR) research focuses on the development of methods which use raw CT scan data. All these studies can be classified into two categories: methods which use the filtered back projection algorithm to reconstruct the image and others which try to avoid this technique so as not to obtain artifacted images caused by its drawbacks. We will now consider 
some of these studies.

In the first category, Rohlfing et al. [1] compare some of these techniques and discuss the problematic issues around MAR. Watzke et al. [2] combine two MAR methods previously proposed: linear interpolation (LI) of the reprojection of metallic objects and multi-dimensional adaptive filtering (MAF) of the raw data. The LI algorithm [3] consists of reconstructing a preliminary image, reprojecting the threshold-segmented metallic objects, linear interpolating in the raw data domain and reconstructing. The MAF algorithm [4] consists of filtering the significant raw data (sinogram values above a threshold selected by the user). This algorithm reduces artifact noise but has no effect on beam hardening. Therefore, a distance dependent merging technique is used for these algorithms. Yu et al. [5] propose a method inspired by Kalender's work, which includes a mean-shift technique to improve accuracy in the segmentation process and an iterative feedback-based interpolation method. Zhao et al. [6] proposed a wavelet method for MAR. It consists of estimating the metallic set by threshold-segmentation. After this, the scan data can be defined by its scaling and wavelet coefficients and a wavelet multiresolution interpolation can be applied to design an appropriate weighting scheme in order to recover the information affected by the artifact.

In the second category, avoiding the FBP algorithm, Wang et al. [7], consider the CT scan as a deblurring problem and try to solve it by using two iterative approaches: the expectation maximization formula (EM) and the algebraic reconstruction technique (ART). The method minimizes iteratively the discrepancy between the measured raw data and the computationally synthesized data to obtain iterative improvements in the retroprojected image. In a similar way, Murphy et al. [8] use an alternating minimization (AM) algorithm to minimize the I-divergence (to maximize the similarity) between the data and its estimation in order to form the image.

All the results of the aforementioned methods are either derived from the raw data obtained from the tomograph, or use it in some way to replace the FBP data. But in most applications, as ours (an intelligent system for designing, simulating and flexible manufacturing of implant-supported dental prostheses), the raw data is not available and experts must infer conclusions from the artifacted FBP images. A new point of view is needed to improve the image quality in this context.

With this consideration in mind, Sohmura et al. [9] replace the artifacted teeth with the $\mathrm{CT}$ representation of a dental cast previously registered. It implies having a patient's accurate cast, CT scan with the markers used for 
the registration and high accuracy at the registration process. As well with the aim of 3D reconstruction, Tognola et al. [10] segment the mandibular surface after enhancing the image. This process consists of a histogram equalization followed by a thresholding. Naranjo et al. [11] transform the image into the polar domain before the processing step. This way, the radial pattern of the artifact is reshaped to a vertical pattern, which makes possible applying a morphological filter. However, the study requires further testing, automation and more extensive validation. This paper extends this study and tries to overcome its lacks.

Regarding automation, the focus of the transformation is estimated without human interaction even in cases of multiple artifact sources. Regarding the processing, the current paper discusses different morphological filters and, in addition, the size of the structuring elements used are estimated depending on the distance to the artifact origin. Finally, a new validation which compares the processed images with a groundtruth set is presented.

Hence, our aim is to describe a new approach in MAR techniques always oriented to 3D reconstruction, which starts with FBP images stored in DICOM files and tries to make medical examination easier and more accurate without additional information.

\section{Method}

\subsection{Morphological filtering}

Mathematical morphology is a theory based on minimum and maximum operations [12,13], constructed from two basic operators. The dilation (eq 1) computes the maximum gray level of a neighborhood determined by a structuring element (SE). On the contrary, the erosion (eq 2) computes the minimum gray level of a neighborhood determined by the transposed structuring element.

$$
\begin{aligned}
& \delta_{B}(f)=\sup \left\{f_{b}, b \in B\right\} \\
& \varepsilon_{B}(f)=\inf \left\{f_{b}, b \in B^{\top}\right\}
\end{aligned}
$$

Combining the dilation and erosion it is possible to define two new operations. The morphological opening (eq 3), consists of an erosion followed by a dilation. The opening of a grayscale image $f$ with the structuring element $B$, $\gamma_{B}(f)$, removes clear areas in the image where the structuring element does 


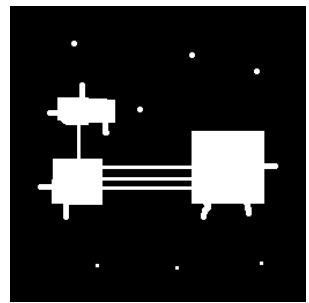

a)

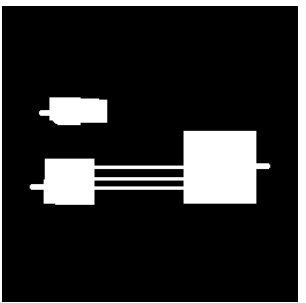

b)

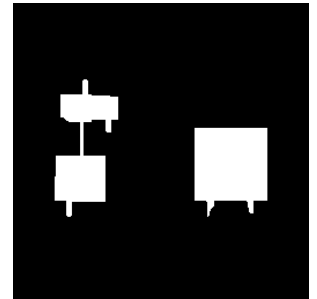

c)

Figure 2: a) Original image. b) Opening with horizontal SE with size $=15$. c) Opening with a vertical $\mathrm{SE}$ with size $=15$.

not fit. On the other hand, the closing (eq 4) consists of a dilation followed by an erosion. In the same way, the closing removes those dark image areas where the structuring element does not fit.

$$
\begin{aligned}
& \gamma_{B}(f)=\delta_{B}\left(\varepsilon_{B}(f)\right) \\
& \varphi_{B}(f)=\varepsilon_{B}\left(\delta_{B}(f)\right)
\end{aligned}
$$

Morphological filtering is a kind of non linear filtering widely used in image noise reduction and artifact elimination [14]. The effects produced by these filters depend on the nature of the filter and on the shape and size of the SE, which fix the analysis surroundings. A wide family of morphological filters has been developed combining the opening and closing operators [15].

Figure 2 shows the effects of the opening of an image using different SEs (horizontal in 2.b) and vertical in 2.c). The clear areas where the SE does not fit are erased (horizontal narrower areas than the SE in b), and vertical narrower in c)).

\subsubsection{Types of morphological filters}

The aim of this study is to reduce the noisy artifacts while preserving, as much as possible, the original image structures to facilitate the 3D reconstruction. In order to achieve this goal, different morphological filters have been tested to select the best option to restore the original image. These filters are:

- Opening-Closing filter: the opening of the original image followed by a closing operation. Analytically: 


$$
O C_{\lambda}(f)=\varphi_{B_{\lambda}} \gamma_{B_{\lambda}}(f),
$$

where $\lambda$ is the SE size.

- Closing-Opening filter: the dual operator of the previous one. Here the operations are implemented in inverse order.

$$
C O_{\lambda}(f)=\gamma_{B_{\lambda}} \varphi_{B_{\lambda}}(f) .
$$

- Alternating sequential filter: concatenation of opening and closing operators where the size of the SE increases from 2 to $\lambda$. Two different filters can be obtained depending on the first operator:

- Starting by opening

$$
A S F O_{\lambda}=\varphi_{B_{\lambda}} \gamma_{B_{\lambda}} \varphi_{B_{\lambda}-1} \gamma_{B_{\lambda-1}} \ldots \varphi_{B_{2}} \gamma_{B_{2}}(f) .
$$

- Starting by closing

$$
A S F C_{\lambda}=\gamma_{B_{\lambda}} \varphi_{B_{\lambda}} \gamma_{B_{\lambda}-1} \varphi_{B_{\lambda-1}} \ldots \gamma_{B_{2}} \varphi_{B_{2}}(f) .
$$

- Closing-Opening Average: the average of the results of the closing and opening operators:

$$
C O A_{\lambda}=\left(\gamma_{B_{\lambda}}(f)+\varphi_{B_{\lambda}}(f)\right) / 2
$$

In section 3 a comparison of these morphological filters is presented (figure 10). In accordance with the results obtained for a test set, consisting of 52 axial CT slices from 20 different patients, the filter with the best performance is implemented in the algorithm.

\subsubsection{Structuring element topology}

The results of the morphological filters are associated not only with the type of filter but also with the correct selection of the shape and size of SE.

As it is shown in figure 2, in order to remove objects with a particular orientation, it is necessary to apply the morphological opening of the image with a SE orthogonally oriented to them. In the case of streaking artifacts (rays emerging from metallic objects), a set of SE orthogonally oriented to 
each ray should be used. This fact makes the process mostly unattainable and for this reason it is necessary to find another way to tackle the problem.

Our approach is based on converting the image from the Cartesian into the polar domain, using the artifact source as the focus of the transformation. With this approach, the radial artifacts go along the angular axis with the same orientation, that is, the streaking rays are transformed into vertical rays and therefore, it is possible to use a single orientation for the SEs for the whole image, orthogonally oriented to the vertical rays [16]. In section 2.2 the geometrical transformation to convert the image into the polar domain is described.

Figure 5 shows the image in figure 1 in the polar domain. As shown, the streaking artifacts are converted into vertical rays, easily removable using a morphological filter with a horizontal SE.

The size of the SE is critical. A large SE will remove significant artifacts but will also blur the image. The larger the SE is, the wider the streaking artifacts it can reduce, but the more blurred the image results. Since the rays are thicker at the metallic object surroundings and become thinner with the distance, a distance adaptive variation of the SE size is proposed and shown in figure 3.

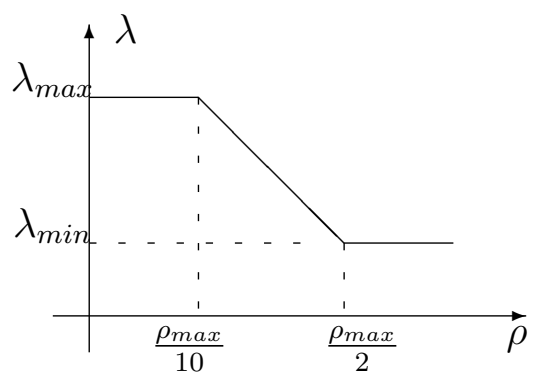

Figure 3: Function of SE size variability.

\subsection{Polar coordinate system}

Images obtained by FBP of CT scan data often show some degree of symmetry since the image is derived from the scattering values of the X-ray beam rotating around the object. Therefore, it is intuitive to define any point in terms of angles and distance, which implies using trigonometric formulae in the rectangular coordinate system. Easier expressions are possible in the polar coordinate system. 
The polar coordinate system determines each point by its radial and angular coordinates denoting the distance from the pole, the origin of symmetry, and the angle defined with the polar axis. Figure 4 shows this relationship.

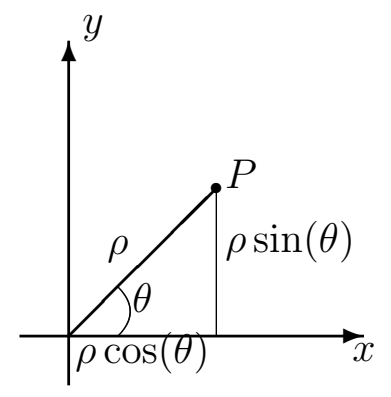

Figure 4: Rectangular-polar coordinate system relationship.

\subsubsection{Definition}

Let $P$ be a point in a two-dimensional coordinate system. We denote $P(x, y)$ to refer to it in rectangular (or Euclidean or Cartesian) coordinates. The transformation into polar coordinates is defined by the following equations:

$$
\begin{array}{r}
\rho=\sqrt{\left(x-x_{c}\right)^{2}+\left(y-y_{c}\right)^{2}}, 0 \leq \rho \leq \rho_{\max } \\
\theta=\arctan \left(\frac{y-y_{c}}{x-x_{c}}\right), 0 \leq \theta \leq 2 \pi
\end{array}
$$

Defining $\left(x_{c}, y_{c}\right)$ as the origin of the streaking rays, that is, taking the streaking source as the focus of the transformation, the conversion of the artifacted image results in the polar image in figure 5 .

In order to automate the whole process, a method to detect the transformation center, $\left(x_{c}, y_{c}\right)$, using granulometry, has been developed and is explained in the following section.

\subsubsection{Automated streaking origin detection}

Granulometry is a geologic term adopted in textured image processing to refer to the extraction of sets of elements with some specific properties in an image [12]. The image is processed by a filtering scheme so that in each step some structures are extracted and refined. 


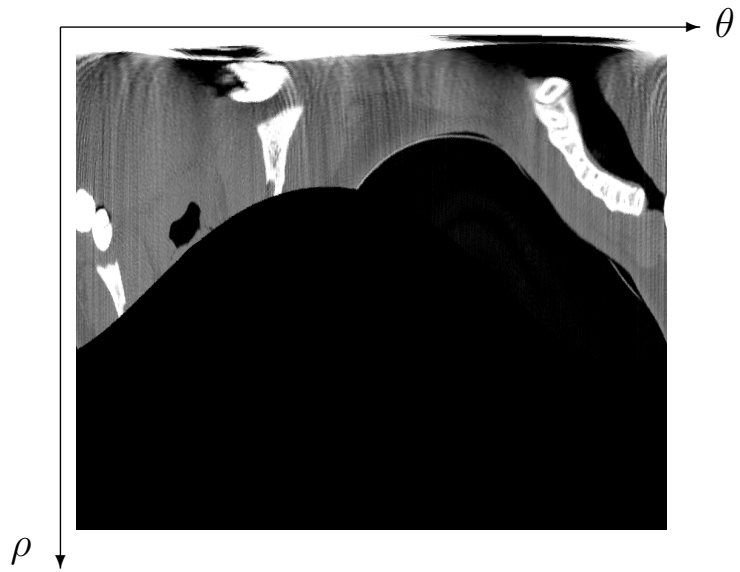

Figure 5: Image of figure 1 in the polar domain.

The strategy is conceptually based on multiple sieves which progressively filter out different parts of the original image. In our case, these sieves are morphological operators with well-known properties, so the design of the granulometry process consists of finding the correct operators to extract the target structures of the image.

Analytically, let $B$ be a convex structuring element and $r, s$ scalar parameters with $r>s>0$.

Consider the opening of a binary image $A$ by $B$ with different sizes.

It follows that:

$$
A \circ r B \subseteq A \circ s B
$$

Then, the granulometry of generator B is a family of morphological operations, $\Psi_{t}^{B}$ for $t \geq 1$

$$
\Psi_{t}^{B}(A)=A \circ t B
$$

It is the set of operators which extract image objects with some desired features, combining different SEs $(B)$ and their sizes $(t)$. Our aim is to detect the streaking source, which intuitively can be defined as the intersection of the family of functions described by the set of streaking lines. So, to deduce these functions, the streaking lines must be extracted, and the scheme shown in figure 6 is designed for this purpose.

First of all, the image is thresholded to extract the binary objects (blobs), which matches the streaking rays. The top-hat openings extract the blobs nearly perpendicular to the structuring elements (SE orientations of $45^{\circ}$ and 


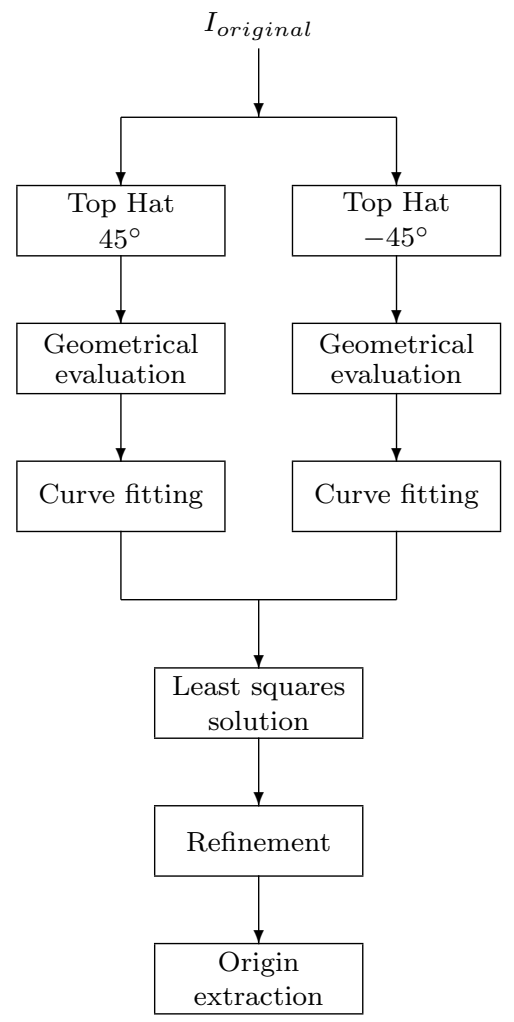

Figure 6: Block diagram of the proposed MAR method.

$-45^{\circ}$ ); that is, those objects with 135 and 225 degrees orientation, respectively. Then, the area, eccentricity and orientation values are computed for all the blobs in the resulting images, and are constrained to reach some values. To sum up, a blob is considered to be a line structure if:

- Area $\geq 20$

- Eccentricity $\geq 0.96$

- Orientation $\in \begin{cases}{\left[105^{\circ}, 165^{\circ}\right]} & \text {, if } \angle_{S E}=45^{\circ} \\ {\left[195^{\circ}, 255^{\circ}\right]} & \text {,if } \angle_{S E}=-45^{\circ}\end{cases}$

Once these geometrical properties are evaluated and the blobs are filtered, we proceed to the curve fitting step. We use a first degree polynomial equation to fit the lines, so we arrive to a system with many more equations than unknowns, that is, an overdetermined system which can be easily solved by the least squares method in the following way: 
$y=A x+b$
where $A=\left(\begin{array}{c}a_{1} \\ a_{2} \\ \vdots \\ a_{n}\end{array}\right)$ and $b=\left(\begin{array}{c}b_{1} \\ b_{2} \\ \vdots \\ b_{n}\end{array}\right)$ are the coefficients of the polynomial expressions of the family of $\mathrm{n}$ lines. Consequently, the solution of the system denoted by $A x=b$ is given by least squares

$$
\widehat{x}=\left(A^{t} A\right)^{-1} A^{t} b
$$

Finally, we carry out a refinement of the data to improve the accuracy of the results. As the first approach is satisfactory due to the previous geometrical filtering, we can remove the outliers of the distribution simply determining if the distance in a coordinate, fixing the other coordinate, is greater than a threshold (that is, those lines which are too far from the estimated center) and redefining the system. This is done for both coordinates. Figure 7 shows this process. 


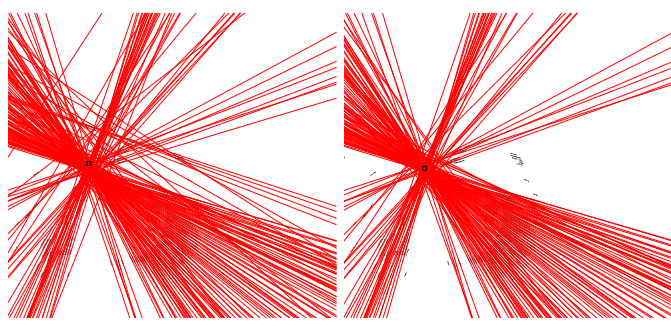

a)

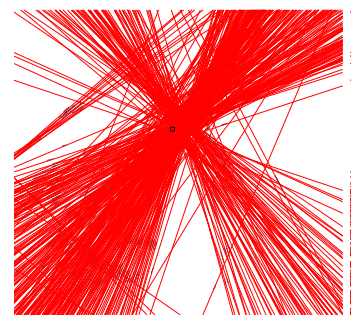

d)

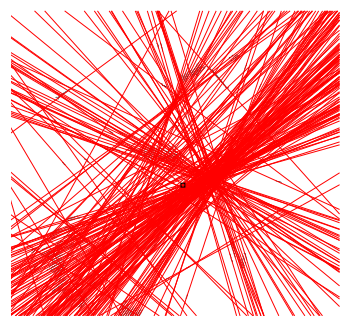

g) b)

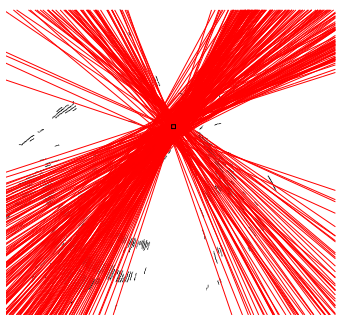

e)

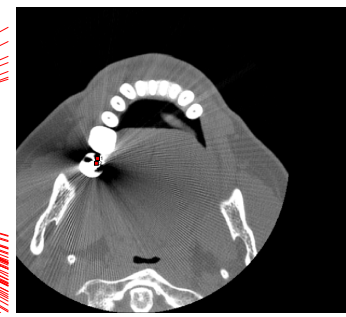

c)

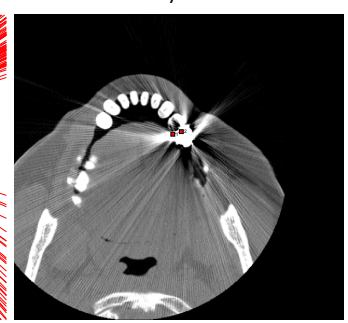

f)

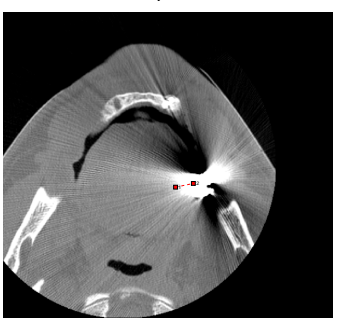

i)

Figure 7: a), d), g): Resulting overdetermined system. b), e), h): The overdetermined system after refinement. c), f), i): Results over the original image.

\section{Multiple metallic objects case}

In situations where there are multiple streaking sources due to the presence of several metallic objects, the human intervention is required in order to achieve the same precision. In these cases, a bounding box must be defined around each origin. The boundary fixes the region of interest (ROI) of each system in such a way that each origin could be processed as a single source case. Finally, the systems are solved by the least squares method to obtain the results shown in figure 8.

\subsection{The algorithm}

The block diagram of the algorithm which synthesizes the whole process explained above is shown in figure 9 . The original image is converted from the 


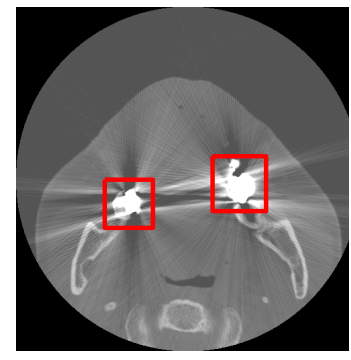

a.)

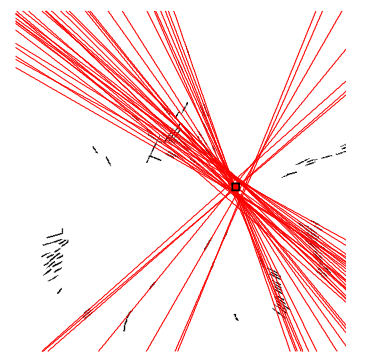

c)

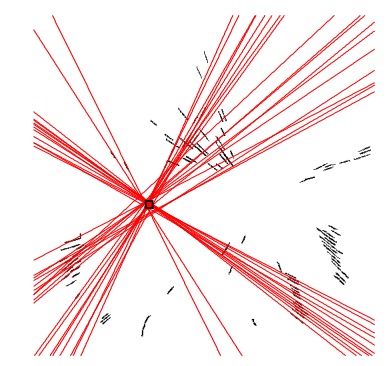

h)

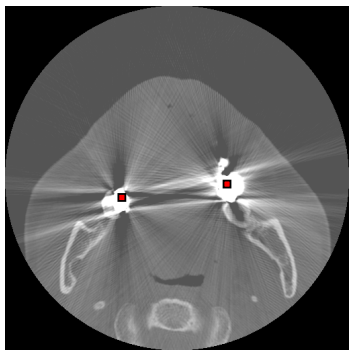

d)

Figure 8: a): Selected origins. b), c): Resulting overdetermined systems for both origins. d): Results over the original image.

Cartesian into the polar domain by means of the polar block. After this, the polar image is filtered using a morphological filter as described in section 2.1, and the result is reconverted into the Cartesian domain, resulting in the filtered image $I_{\text {filtered }}$. On the other hand, the original image is segmented in order to detect the cavities $\left(I_{\text {original }}<T\right)$ using a simple threshold. The threshold block generates a mask $\left(I_{m s k}\right)$ where the pixels corresponding to the cavities are set to 1 and the rest of the pixels are set to 0 . The cavities are also artifacted but due to their low density values, they are easily reconstructible, hence they are preserved from the effects of the filter. The output image is obtained by merging the original and the filtered images in the following way:

$$
I_{\text {output }}=I_{\text {original }} \times I_{m s k}+I_{\text {filtered }} \times\left(1-I_{m s k}\right)
$$

In the multiple origin case the method consists of performing the same process for every streaking source. That is, performing different transformations (one for each streaking origin), filtering and transforming again the 


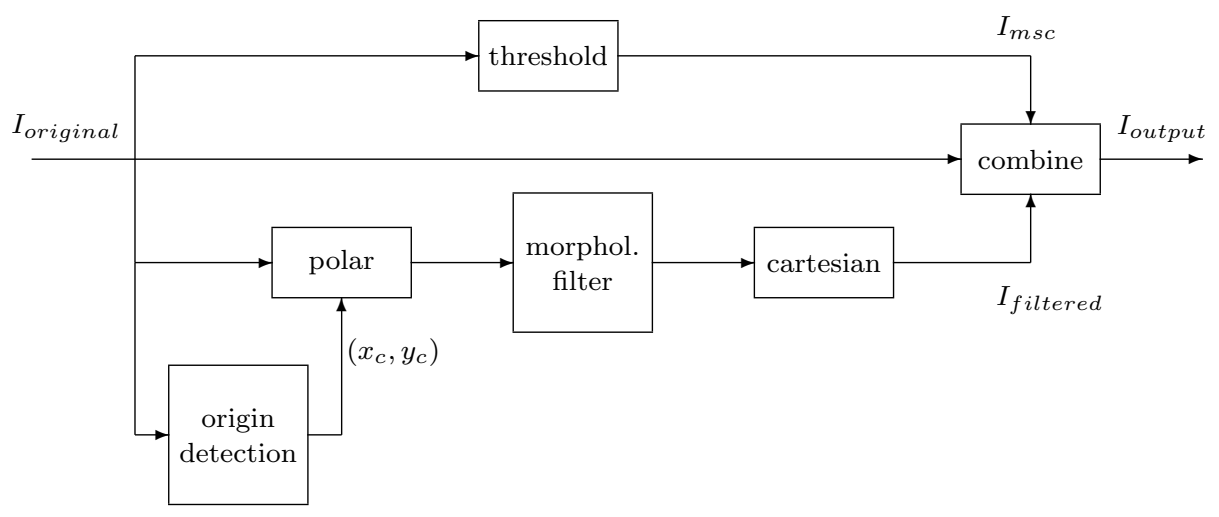

Figure 9: Block diagram of the proposed MAR method.

processed images. Finally, $I_{\text {filtered }}$ is computed averaging all the filtered images.

\section{Results}

In this section the results of the MAR method presented in this paper are shown. 52 slices from 20 different patients, whose data sets have been obtained using different tomographs (GE MEDICAL SYSTEMS HiSpeed QXi and Philips Medical Systems - Philips CT Aura), have been considered in order to evaluate the method, shaping the test set. In order to analytically evaluate the performance of the method the test set has been manually segmented by a group of experts, defining the groundtruth set. Hence, the test set has been processed by the presented method and the resulting images have been compared with the groundtruth set by means of Jaccard index (JC) and Dice coefficient (DC). The cavities have been segmented using a threshold parameter $T=-500 \mathrm{HU}$ for the whole evaluation and yield successful results. It is necessary to emphasize that the cavities are easily isolated from beam hardening because this artifacts appears in the surroundings of the teeth. This section shows the justification of the selection of both the filter and the size of the SE. Once these parameters are fixed, the method is evaluated.

\subsection{Filter selection}

The filters in section 2.1 have been tested, fixing the size of the SE, and the results for an example slice are shown in figure 10. Although the output 
images are similar, the $C O A_{\lambda}$ filter has been proven to give the best results with regard to $3 \mathrm{D}$ reconstruction. Table 3.1 shows the resulting values. In all cases, the size of the SE has been set to $\lambda=9$.

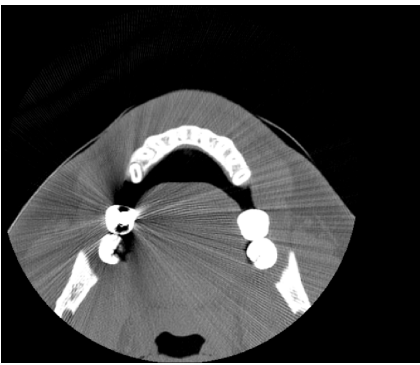

a)

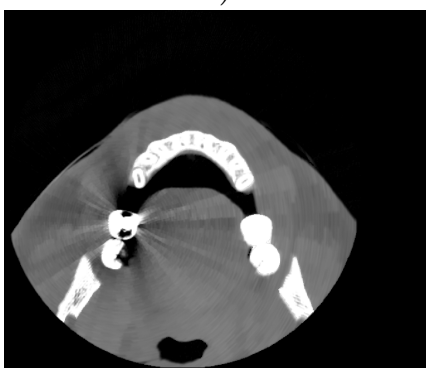

d)

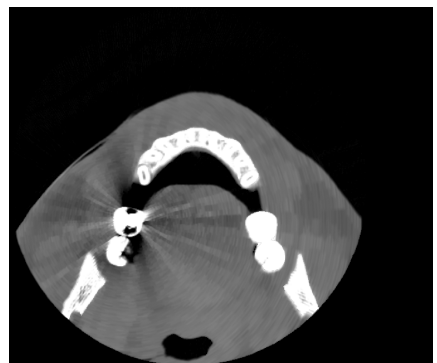

b)

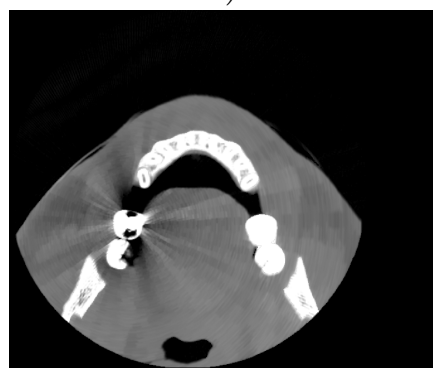

e)

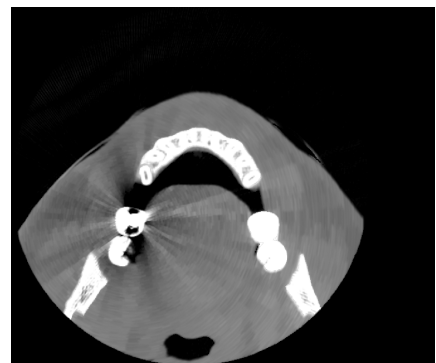

c)

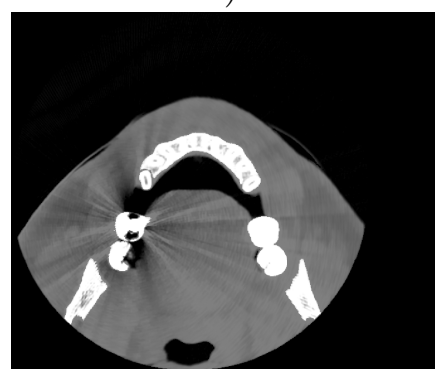

f)

Figure 10: a) Original image; Images filtered using b) $O C_{\lambda}$ filter; c) $C O_{\lambda}$ filter; d) $A S F O_{\lambda}$ filter; e) $A S F C_{\lambda}$ filter; f) $C O A_{\lambda}$ filter. In all cases the size of the $\mathrm{SE}$ is $\lambda=9$.

\begin{tabular}{|l|l|l|}
\hline Filter type & JC improvement & DC improvement \\
\hline$O C_{\lambda}$ & 0.1617 & 0.1074 \\
\hline$C O_{\lambda}$ & 0.1608 & 0.1067 \\
\hline$A S F O_{\lambda}$ & 0.1674 & 0.1131 \\
\hline$A S F C_{\lambda}$ & 0.1893 & 0.1146 \\
\hline$C O A_{\lambda}$ & 0.1927 & 0.1159 \\
\hline
\end{tabular}

Table 1: Mean improvements in $\mathrm{JC}$ and $\mathrm{DC}$ achieved with filters a) $O C_{\lambda}$; b) $C O_{\lambda} ;$ c) $A S F O_{\lambda} ;$ d) $A S F C_{\lambda}$; e) $C O A_{\lambda}$. 


\subsection{Structuring element size selection}

Once the filter type is selected( $C O A_{\lambda}$ filter), the size of the SE needs to be tested following our objectives of reducing the artifacts as much as possible (while also preserving the structures) to improve the $3 \mathrm{D}$ reconstruction. As shown in figure 11, the larger the $\mathrm{SE}$ is, the harder artifacts it can reduce but the more blurred the image results. In conclusion, a large SE is needed $(\lambda>20)$ to reduce streaking in the area surrounding the metallic object, but this selection produces significant degradation, mainly in outer tissues. This effect is noticeable in the areas highlighted in figure 11). To overcome this problem a distance adaptive size of the SE is used, as explained in section 2.1. A comparison of the images obtained using different adaptive sizes for the same example slice is shown in figure 11.

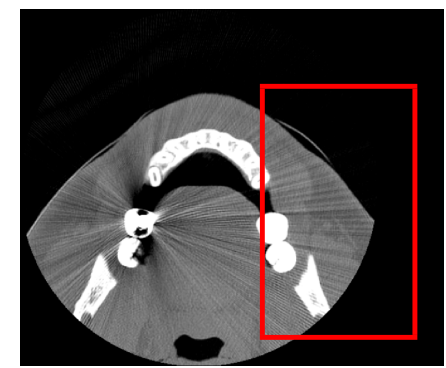

a)

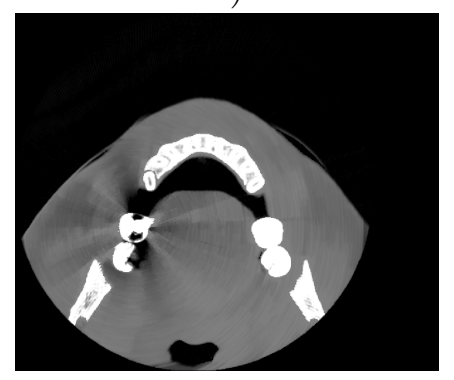

d)

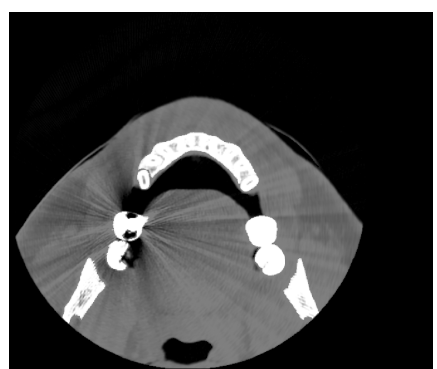

b)

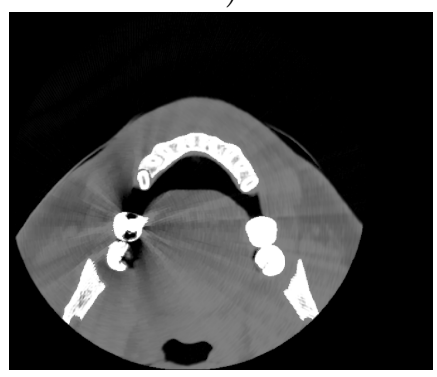

e)

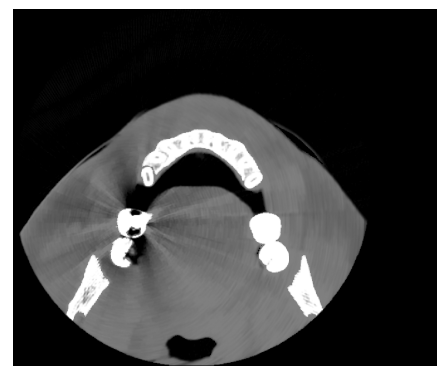

c)

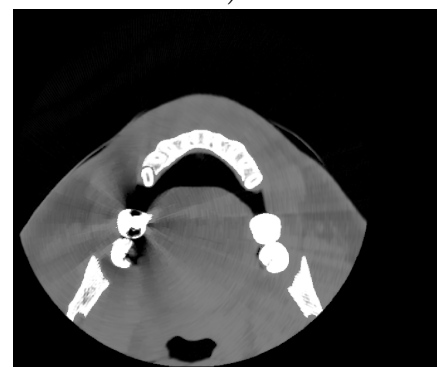

f)

Figure 11: a) Original image; Image 11 filtered using the $C O A_{\lambda}$ filter with b) fixed $l a m b d a=5$; c) fixed lambda $=15$; d) fixed lambda $=25$ e) variable size from $\lambda_{\min }=3$ to $\lambda_{\max }=15$; e) variable size from $\lambda_{\min }=3$ to $\lambda_{\max }=25$.

Best result is obtained through adaptive size from $\lambda_{\min }=3$ to $\lambda_{\max }=25$. Table 3.2 shows the resulting values. 


\begin{tabular}{|l|l|l|}
\hline SE size & JC improvement & DC improvement \\
\hline$\lambda=5$ & 0.1920 & 0.0995 \\
\hline$\lambda=15$ & 0.1933 & 0.1266 \\
\hline$\lambda=25$ & 0.2018 & 0.1345 \\
\hline$\lambda_{\min }=3$ to $\lambda_{\max }=15$ & 0.2401 & 0.1770 \\
\hline$\lambda_{\min }=3$ to $\lambda_{\max }=25$ & 0.2649 & 0.1956 \\
\hline
\end{tabular}

Table 2: Mean improvements in JC and DC achieved by means of $C O A_{\lambda}$ with a) $\lambda=5$; b) $\lambda=15$; c) $\lambda=25$; d) $\lambda_{\min }=3$ to $\lambda_{\max }=15$; and e) $\lambda_{\min }=3$ to $\lambda_{\max }=25$.

\subsection{Process evaluation}

Experimental results have shown that the configuration $C O A_{\lambda}$ filter with variable $\lambda$ (from $\lambda_{\min }=3$ to $\lambda_{\max }=25$ ) gives the best performance among the tested options. For this reason, it is consolidated as the configuration used in the MAR method presented. Figures 12 and 13 show the results obtained for different patients with single and multiple origin cases respectively. 

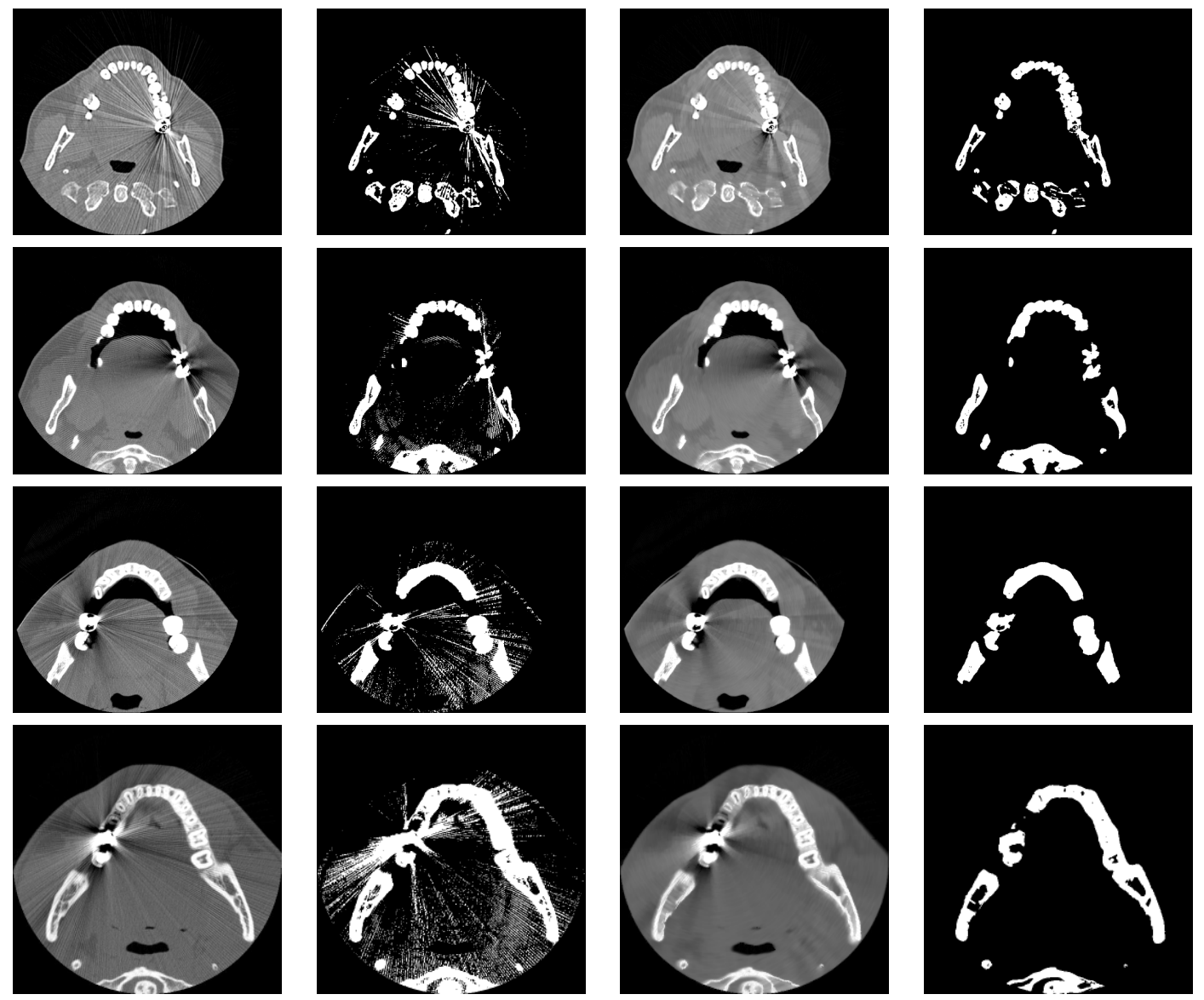

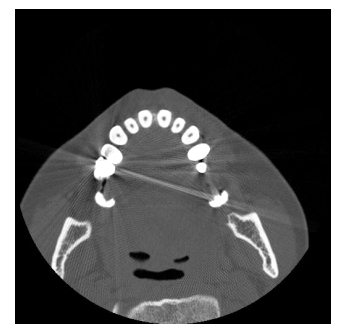

a)

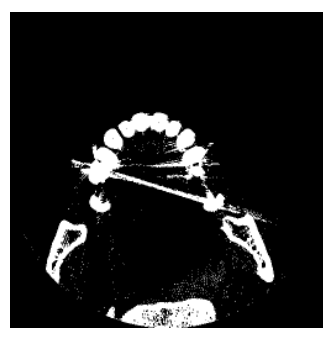

b)

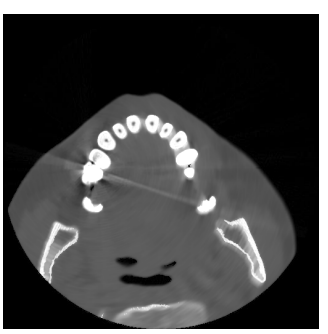

c)

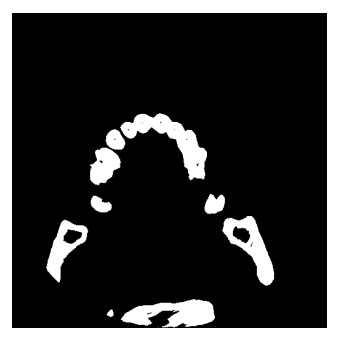

d)

Figure 12: a) Original images. b) Original images thresholded. c) Processed images. d) Processed images thresholded. 

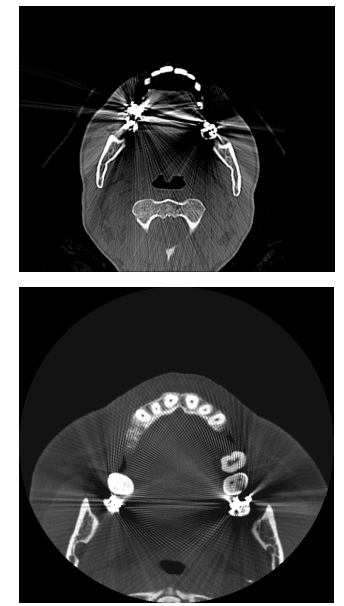

a)
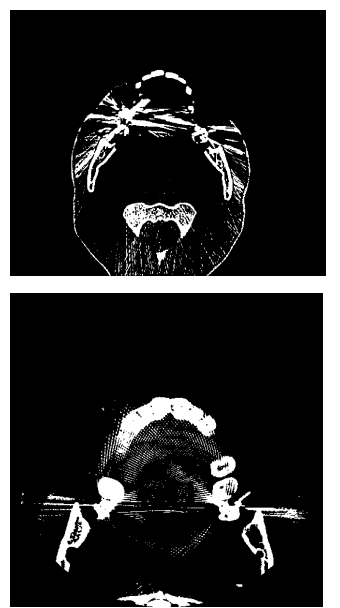

b)
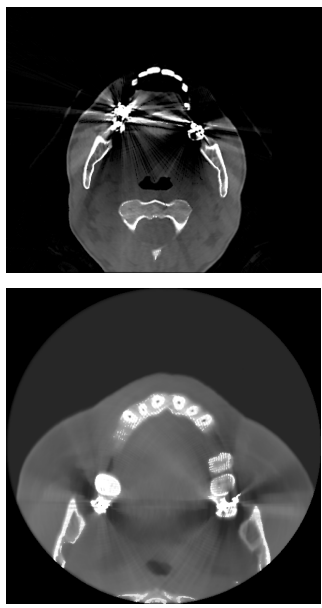

c)
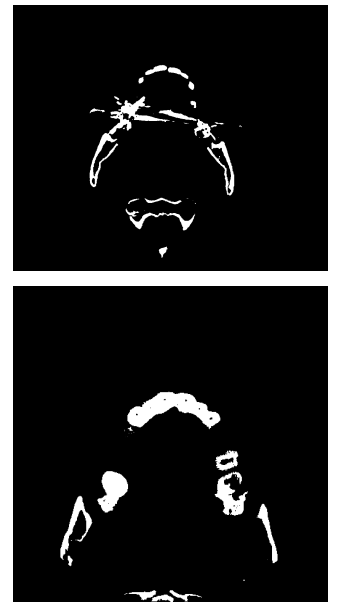

d)

Figure 13: a) Original images. b) Original images thresholded. c) Processed images. d) Processed images thresholded.

It can be seen that the method reduces significantly streaking artifacts and noise, even in cases with heavy degradations, preserving to a great extent teeth, bone, cavities and soft tissues.

The resulting images have been analyzed obtaining a mean improvement in JC and DC of 0.2649 and 1956 respectively, in cases with single metallic object, and 0.1966 and 0.1747 , in cases with multiple metallic objects.

Finally, figure 14 shows a comparison between the proposed method and other image denoising algorithms: Donoho's digital wavelet (DWT) $[17,18]$ and discrete cosine transform (DCT) based methods [19]. 


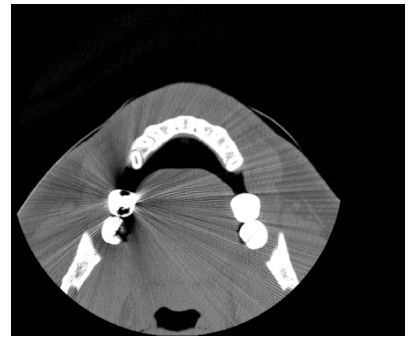

a)

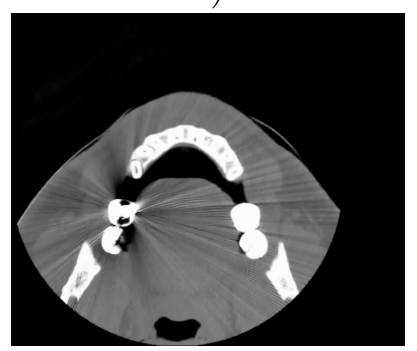

c)

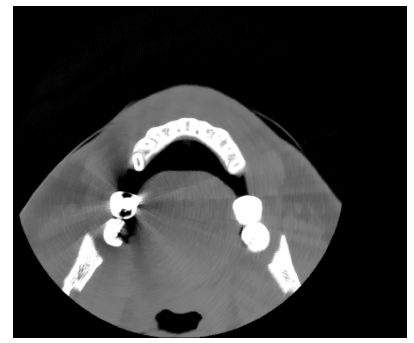

b)

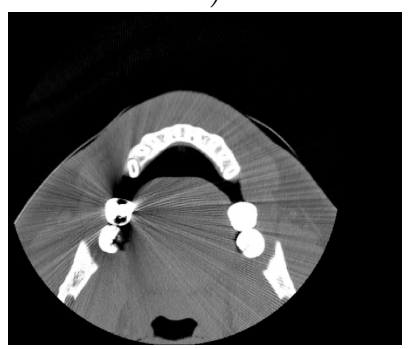

d)

Figure 14: a) Original image. Image processed by b) proposed method; c) DCT-based method; d) DWT method.

The proposed method has given the best results since it has been developed in order to reduce this specific noise pattern, achieving an improvement in Jaccard similarity coefficient of 0.2649 versus 0.1296 and 0.028 obtained by DWT and DCT methods respectively.

As it has been commented previously, most of the commercial applications threshold the image to define the structure to reconstruct. Figure 12 shows the algorithm input images and the result of thresholding them (with a threshold grayscale value of 130), and the same for the processed ones.

The original dental images are remarkably affected by noise and strong artifacts such as beam hardening and streaking, what makes impossible to isolate hard tissues for 3D reconstruction. The processed images are sensibly less affected by these effects, so this fact makes the reconstruction easier. For all the studied cases (52 axial CT slices from 20 different patients) the algorithm parameters, the size of the SE used in the morphological filter and the threshold value, have been set to $\lambda_{\min }=3, \lambda_{\max }=25$ and $\mathrm{T}=-500 \mathrm{HU}$, respectively.

Resulting images show how the method reduces significatively streaking artifacts and noise, not smoothing teeth and preserving bones and cavities. 
However, the beam hardening surounding the metallic object is not relevantly reduced because the suitable size of SE to remove streaking artifacts is not large enough to erase this other effect. A wider SE would over-smooth the rest of the image. To solve this drawback new ideas are being studied and future works will feature this improvement. The processing time of the algorithm on MatLabßhas been about 1 second for a $512 \times 512$ pixel image in a worstcase scenario using an ordinary PC (Pentium IV at $2.8 \mathrm{MHz}$ and $1 \mathrm{~GB}$ of RAM).

Finally, figure 15 shows the improvement of the reconstruction of the CT data after having been processed by the presented method.

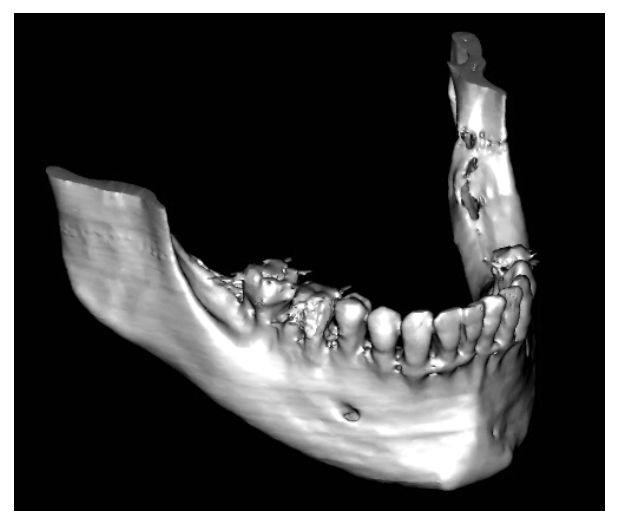

a)

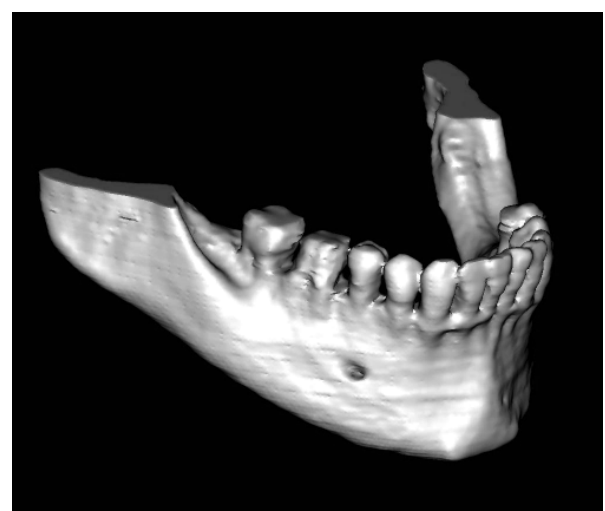

$\mathrm{b}$

Figure 15: 3D jaw reconstruction from original (a) and post-processed CT data (b).

\section{Discussion}

In this paper a new method for metallic artifact reduction has been presented. This method, based on mathematical morphology in the polar domain, has been automated and has proven to reduce streaking and noise artifacts, while causing very little smoothing of the anatomic structures, even in multiple origin artifact cases. One of the contributions of the presented method is the use of CT images as inputs as oppose to all other MAR methods which use projection data. This condition is crucial in those applications where the raw data is not available. The method is particularly efficacious in reducing the streaking artifacts, which are the major cause of distortion in the $3 \mathrm{D}$ reconstruction, so it is specially suitable for enhancing these views. 
In addition, due to its low computational cost, the method is also suitable for real time applications. The method has been compared with other image denoising algorithms achieving good results.

However, the method also presents some weaknesses. The improvement achieved is bounded to the quality of the reconstructed CT image and to the morphological filter used. For this reason, the method is limited to slightly artifacted images. Its performance decreases when multiple metallic objects are present in the same slice, and over all when these objects are close. Beam hardening is hardly reduced since it presents a wide pattern and very low values.

Nevertheless, the method has applicability to a wide range of applications, specially in those oriented to 3D reconstruction. Furthermore, the presented algorithm can also be used as a previous stage for other medical imaging applications or other MAR methods.

Future work will focus on implementing a new method (based on the algorithm described) able to process a full CT study considering the information of adjacent slices to enhance the 3D reconstruction of the scanned object.

[1] T. Rohlfing, D. Zerfowski, J. Beier, N. Hosten, P. Wust, R. Felix, Reduction of metal artifacts in computed tomographies for planning and simulation of radiation therapy., Computer Assisted Radiology and Surgery (1998) 57-62.

[2] O. Watzke, W. Kallender, A pragmatic approach to metal artifact reduction in CT: merging of metal artifact reduced images, European Radiology 14 (2004) 849-856.

[3] W. Kalender, R. Hebel, J. Ebersberger, Reduction of CT artifacts caused by metallic implants., Radiology 164 (1987) 576577.

[4] M. Kachelriess, O. Watzke, W. Kalender, Generalized multi-dimensional adaptive filtering (MAF) for conventional and spiral single-slice, multislice and cone-beam CT., Med. Phys. 28 (2001) 457490.

[5] H. Yu, K. Zeng, G. Bharkhada, D.and Wang, M. Madsen, O. Saba, B. Policeni, W. Howard, M.and Smoker, A segmentation-based method for metal artifact reduction, Academic Radiology 14 (4) (2007) 495-504. 
[6] Z. Shiying, T. Kyongtae, B. Whiting, G. Wang, A wavelet method for metal artifact reduction with multiple metallic objects in the field of view, Journal of X-Ray Science and Technology 10 (2002) 67-76.

[7] G. Wang, D. Snyder, J. O'Sullivan, M. Vannier, Iterative deblurring for CT metal artifact reduction, IEEE Transactions on Medical Imaging 15 (5) (1996) 657-664.

[8] R. Murphy, D. Snyder, D. Politte, J. O'Sullivan, A sieve-regularized image reconstruction algorithm with pose search in transmission tomography, in: SPIE Medical Imaging 2003: Image Processing conference, 2003.

[9] T. Sohmura, H. Hojoh, N. Kusumoto, M. Nishida, K. Wakabayashi, J. Takahashi, A novel method of removing artifacts because of metallic dental restorations in 3-d ct images of jaw bone, Clinical Oral Implant Research 16 (2005) 728735.

[10] G. Tognola, M. Parazzini, G. Pedretti, P. Ravazzani, F. Grandori, A. Pesatori, M. Norgia, C. Svelto, Novel 3d reconstruction method for mandibular distraction planning, International Workshop on Imaging Systems and Techniques (2006) 82- 85.

[11] V. Naranjo, R. Llor'ens, P. Paniagua, M. A. niz, S. Albalat, A new approach in metal artifact reduction for ct $3 \mathrm{~d}$ reconstructionl, Journal of Periodontology 75 (2) (2004) 322-326.

[12] J. Serra, Image analysis and mathematical morphology, Academic Press, London, 1982.

[13] J. Serra, Image analysis and mathematical morphology, vol. II: Theoretical advances, Academic Press, London, 1988.

[14] V. Naranjo, A. Albiol, J. Mossi, A. Albiol, Morphological lambdareconstruction applied to restoration of blotches in old films, in: 4th IASTED International Conference on Visualization, Imaging and Image Processing, 2004.

[15] J. Serra, L. Vincent, An overview of morphological filtering, Circuits, Systems, and Signal Processing 11 (1) (1992) 47-108. 
[16] M. Luengo-Oroz, J. Angulo, G. Flandrin, J. Klossa, Mathematical morphology in polar-logarithmic coordinates. Application to erythrocyte shape analysis, in: 2th Iberian Conference on Pattern Recognition and Image Analysis (IbPRIA'2005). Lecture Notes in Computer Science, Vol. LNCS 3523, 2005, pp. 199-205.

[17] D. Donoho, Denoising by soft-thresholding, IEEE Trans. Inform. Theory 41 (3) (1995) 613627.

[18] B.-J. Yoon, P. P. Vaidyanathan, Wavelet-based denoising by customized thresholding, in: Acoustics, Speech, and Signal Processing, 2004. Proceedings. (ICASSP '04), Vol. 2, 2004, pp. 925-8.

[19] R. Oktem, L. Yarovslavsky, K. Egiazarian, J. Astola, Transform domain approaches for image denoising, Journal of Electronic Imaging 11 (2) (2002) 149-156. 